\title{
Fuzzy clean, fuzzy green
}

\author{
Subhas Sikdar ${ }^{1}$
}

Published online: 24 June 2017

(C) Springer-Verlag GmbH Germany 2017

Quarter of a century ago, an academic stalwart asked me why, as a matter of rhetoric, for products or processes we used the adjective "clean", but not "cleaner"? The point was immediately conceded, but the question pointed to a fundamentally uncritical affinity that researchers in the environmental fields exhibit for absolute descriptors such as clean, green, and now, sustainable. In reality, we mean cleaner when we say clean, and more sustainable when we say sustainable. The science of metrology can be applied to tease out quantitative values of certain important attributes of products or technologies. These values necessarily place claims of cleanliness on comparative platforms. The metrics used to support claims, however, is rarely transparent. A company called Terra choice ${ }^{1}$ examined 4744 home and family products in 2010 and found that only $4.5 \%$ of the entries are "sin free", i.e. the cleanliness claims were factually justified. The rest of the claims were false or misleading. There are various reasons why these claims are often misleading. An educated guess is that in the main, the temptation to gain an immediate market advantage caused by a cleanliness claim outweighs the ethical need to subject the claim to costly validation. Another possible reason is that there is little consequence of false advertisement, and that since there will be others engaged in inaccurate advertisement, why restrain your own business? In business and news media literature, the words "green", "clean", and "sustainable" are as pliable as they are in science and engineering literature. The spectrum of variations remains unquantified, unarticulated, and ignored.

Subhas Sikdar

subhas.sikdar@gmail.com

1 Cincinnati, OH, USA
Surely no one would object to the idea of quantifying degree of cleanliness. In fact, in the 1980s and 1990s, waste management activity was obsessed with the question: "how clean is clean?". Regulations provided the metric in terms of the extent of reduction or destruction of an offending waste or identified chemical. Designing cleaner or greener processes or products is a voluntary endeavour. Consequently there has been no regulatory requirement on defining cleanliness or measuring degree of cleanliness. It has been up to the technology developers, corporations, professional organizations, and academia to devise metrics that can be used to provide a sense of comparative greenness. Typically, a number of indicators or metrics would be used and the claim of cleanliness would be depended upon an overall measure of superiority derived from the values of the indicators. Despite some recent books on measurement of cleaner, greener, and sustainable technologies, not much effort is seen among researchers on quantitative assessment of cleanliness. $^{2}$ Unfortunately, the process as practiced in industry is not transparent. In academic research, there are some signs of validating claims by the use of metrics. Sometimes a few metrics would be imbedded in process integration tools used by engineers. There is also a disturbing trend towards claiming cleanliness based on one or two indicators. Such claims are not generally justifiable for not considering all applicable attributes of an assessed system. For instance, as the dominant objective in sustainable process design these days is to show reduction in carbon dioxide emission, a design based on this one attribute appears to be

\footnotetext{
${ }^{1} \mathrm{http}: / /$ sinsofgreenwashing.com/ accessed June 2017.

2 Measuring Progress Towards Sustainability: a treatise for engineers, by Sikdar, S, Sengupta, D., and Mukherjee, R., Springer, Switzerland 2017. Bell, S, and Morse, S., Measuring sustainability: learning by doing, Earthscan Publications, London, 2003. Hak, T., Moldan, B., and Dahl, A., Sustainability indicators, Island Press, Wash, DC, 2007.
} 
Table 1 Clean claims based on insufficient metrics

\begin{tabular}{ll}
\hline Main metric used for claim & Systems for which the metric is used \\
\hline Carbon footprints & Chemical processes, catalytic unit processes, energy and renewable fuel systems \\
Ecological footprint & Regional economic systems \\
Recycle/reuse ability & Materials systems \\
Local cleanliness & Automotive, battery storage \\
Substitutability & Components of home appliances, automobiles \\
Water footprints & Biofuels \\
\hline
\end{tabular}

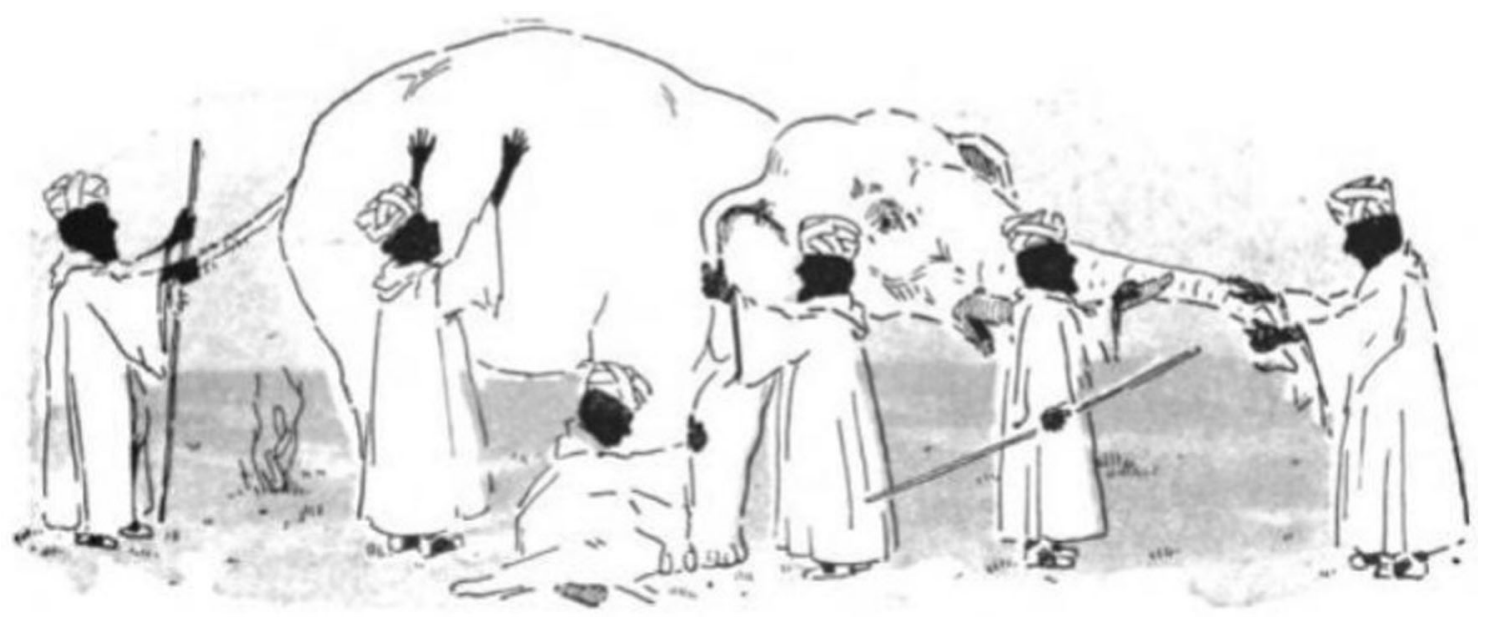

Fig. 1 Claim of superiority is justified when measurements are comprehensive

sufficient in making the claim of superiority. Other examples of where reliance on a single metric is tempting are shown in Table 1.

The problem of comparative assessment of course is not new. The effect of not using an overall measure is well parodied in the description of an elephant by an assembly of blind men who touch only specific parts of the animal and identify it variously based on evidence. ${ }^{3}$ Figure 1 shows the caricature. The American poet Saxe wrote a poem on this theme, a part of which is reproduced here. As the poet says, we can be always partly right while being totally wrong!

And so these men of Indostan

Disputed loud and long,

Each in his own opinion

Exceeding stiff and strong,

Though each was partly in the right,

And all were in the wrong! (John Godfrey Saxe)

Cleanliness is quantitatively assessed only after the process or product has been designed, built, and

\footnotetext{
3 https://en.wikipedia.org/wiki/Blind_men_and_an_elephant, accessed June 2017.
}

performance data collected. Stated differently, this assessment is retrospective. Innovating a new product or process, however, is a prospective activity. It requires the creation of a thing that did not exist before and on which no performance data are available. For sure, some principles of avoiding environmental impacts can be taken advantage of in the design process, but there cannot be a quantitative assessment in the absence of actual performance. Additionally, some impacts cannot be anticipated. Recent history of innovations is replete with examples of unintended consequences of products. Perhaps the most significant example is the use of tetraethyl lead in gasoline. Other examples are shown in Table 2.

Despite the difficulties of predicting or anticipating environmental impact information at the design stage of a product or a process, it is necessary to ask questions that were not asked before. A good place to start would be to examine the various categories of environmental impacts by the components used in a synthesis and by the emissions that are known from the chemistry of the process. Some of these impacts can be assessed, and other impacts are expected but not yet measured (known unknowns). After the advent of life cycle assessment (LCA) methodology, categories of life cycle impacts (LCIs) have been 
Table 2 Failure to anticipate environmental impacts at the front end of innovations
Fig. 2 Environmental impact and value creation of steel products through the life cycle of the manufacture. Adapted from Roland Clift, Dankwerts Lecture 2010, AIChE Mtg, SALT LAKE CITY

\begin{tabular}{lll}
\hline Cases & Benefit & Unintended consequences \\
\hline $\mathrm{Pb}$ in gasoline & Elimination of knock & Air, water, and soil pollution \\
$\mathrm{Pb}$ in bullets & Heavy, malleable & Soil pollution in test ranges \\
Chlorofluorocarbons & Inert refrigerants, cleaners & Stratospheric ozone depletion \\
Asbestos, PCBs & Insulation & Respiratory illness \\
Perchlorohydrocarbons & Cleaning & Groundwater pollution \\
MTBE in gasoline & Oxygenates & Groundwater pollution \\
Chromium in electroplating & Protective to rusting & Groundwater pollution \\
PFOA, PFOS & Non-stick, and slain repellent & Immune compromise \\
\hline
\end{tabular}

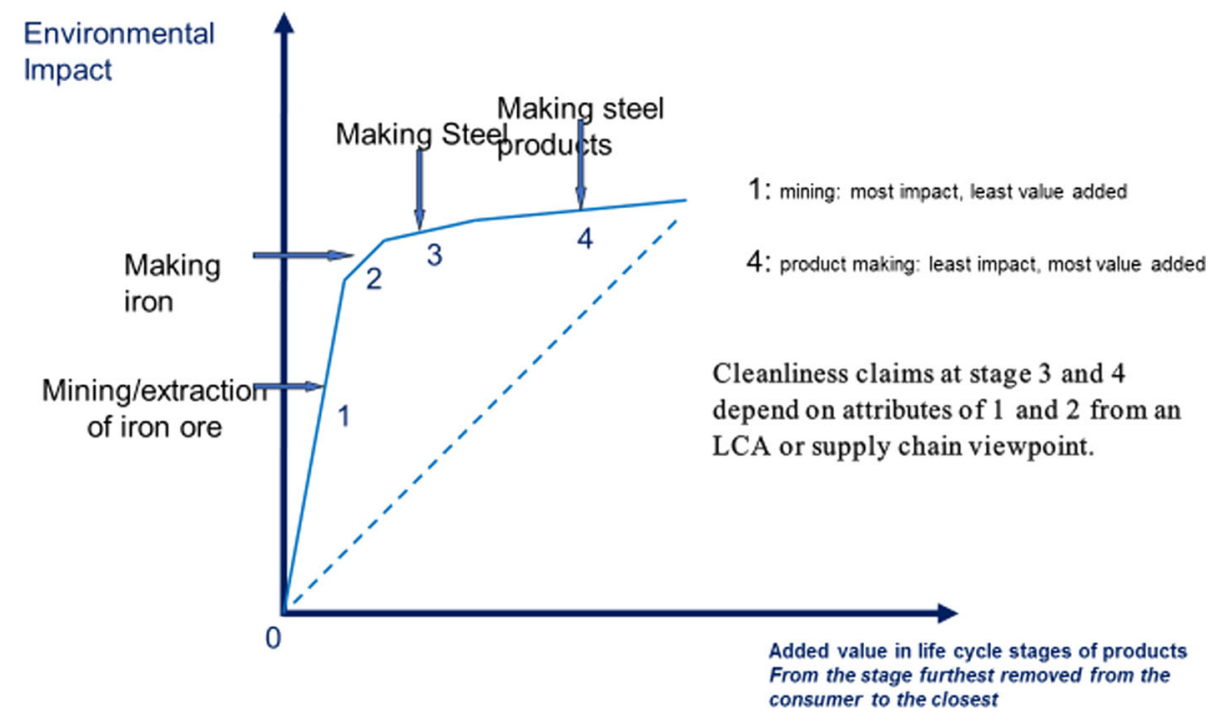

identified. ${ }^{4}$ These impacts should be examined for potential assessment of newer designs. Life cycle stages of products often reveal interesting insights into potential impacts when one focuses on appropriate stages of a product's life cycle from cradle to gate or other assessment constructs. An example of such an analysis is shown in Figure 2. Here each stage of an idealized steel-making process life cycle from mining to a finished product is plotted against the value creation in that stage. It is quite clear from this simple diagram that the greatest environmental impact happens early in the life cycle, i.e. in mining, where value addition is small, compared to much cleaner operations later in the value chain where higher values are added. This realization should instruct us to pay greater scrutiny to potential environmental impacts in the early stages. Overall, the impact assessment of the product in question would have to be based on all relevant indicators that affect the product or process, not just on a fashionable one or two.

Subhas Sikdar

Editor-in-Chief

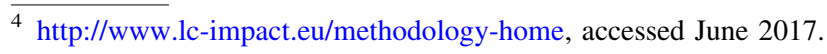

